\title{
IMPROVEMENT OF NAKAMULA'S UPPER BOUND FOR THE ABSOLUTE DISCRIMINANT OF A SEXTIC NUMBER FIELD WITH TWO REAL CONJUGATES
}

\author{
R. J. STROEKER
}

\begin{abstract}
Let $\mathbf{K}$ be the compositum of a real quadratic number field $\mathbf{K}_{2}$ and a complex cubic number field $\mathbf{K}_{3}$. Further, let $\varepsilon$ be a unit of $\mathbf{K}$ which is also a relative unit with respect to $K / K_{2}$ and $K / K_{3}$. The absolute discriminant of this non-Galois sextic number field $\mathbf{K}$ is estimated from above by a simple, strictly increasing, polynomial function of $\varepsilon$. This estimate, which can be used to determine a generator for the cyclic group of relative units, substantially improves a similar bound due to Nakamula. The method employed makes nontrivial use of computer algebra techniques.
\end{abstract}

\section{INTRODUCTION}

In his beautiful paper [2], Nakamula shows great arithmetic skills in the way he calculates fundamental units and class numbers of number fields of absolute degree 6 over $\mathbf{Q}$. In the paper cited, Nakamula considers sextic fields $\mathbf{K}$ with a real quadratic subfield $\mathbf{K}_{2}$ and a complex cubic subfield $\mathbf{K}_{3}$. Or rather, take any quadratic extension $\mathbf{K}_{2}$ of $\mathbf{Q}$ of discriminant $d_{2}>0$ and any cubic extension $\mathbf{K}_{3}$ of $\mathbf{Q}$ of discriminant $d_{3}<0$; then the compositum $\mathbf{K}:=\mathbf{K}_{2} \cdot \mathbf{K}_{3}$ is a non-Galois sextic field of positive absolute discriminant $D$. Consider $\mathbf{K}$ to be embedded in the reals $\mathbf{R}$.

It is the unit structure of $\mathbf{K}$ that interests us. As the torsion subgroup of the unit group is trivial, it is sufficient to consider only the group $E$ of positive units of $\mathbf{K}$. Let $H$ be its subgroup of relative units with respect to $\mathbf{K}_{2}$ and $\mathbf{K}_{3}$, that is,

$$
H:=\left\{\varepsilon \in E \mid \operatorname{Norm}_{\mathbf{K} / \mathbf{K}_{2}}(\varepsilon)=\operatorname{Norm}_{\mathbf{K} / \mathbf{K}_{3}}(\varepsilon)=1\right\} .
$$

Both free unit groups $E_{2}$ and $E_{3}$ of positive units of $\mathbf{K}_{2}$ and $\mathbf{K}_{3}$, respectively, have rank 1, and $E$ has rank 3, by Dirichlet's unit theorem. Consequently, $H$ is an infinite cyclic group. One of the main points of Nakamula's paper is to give an effective way of calculating the three generators of the free unit group $E$, and he succeeds in doing this by expressing the generators of $E$ in terms of the generators of $E_{2}, E_{3}$, and $H$.

Assuming that generators for $E_{2}$ and $E_{3}$ have been found, we concentrate on $H$.

Received by the editor January 12, 1991 and, in revised form, July 3, 1991.

1991 Mathematics Subject Classification. Primary 11R21, 11R29, 11-04. 
Let $\varepsilon \in H, \varepsilon>1$, be given. To ensure that $\varepsilon$ generates $H$, is is sufficient to check that a relation of the form

$$
\varepsilon=\xi^{n} \quad \text { for a } \xi \in H \text { and } n \in \mathbf{N} \text { with } n \geq 2
$$

is impossible. In order to do this, suppose that a strictly increasing function $T: \mathbf{R}^{+} \rightarrow \mathbf{R}^{+}$exists such that for each $\xi \in H$ the inequality $D(\xi)<T(\xi)$ holds, where $D(\xi)$ is the discriminant of $\xi$ with respect to $\mathbf{K} / \mathbf{Q}$. If the inverse $T^{-1}$ of $T$ can be explicitly evaluated at each relevant value, then a useful upper bound for the exponent $n$ in (2) can be obtained as follows. Let $D$ be the absolute discriminant of $\mathbf{K}$. As $\varepsilon>1$, it follows from (2) that

$$
0<D \leq D(\xi)=D(\sqrt[n]{\varepsilon})<T(\sqrt[n]{\varepsilon})
$$

which implies $n<\log \varepsilon / \log T^{-1}(D)$, provided $D>T(1)$. In Lemma 2 of [2] the author uses the function

$$
T(x):=16\left(\left(x+\frac{9}{7}\right)^{7}-290\right)^{2},
$$

to prove (see Theorem 1 of [2])

Theorem 1 (Nakamula). Let $\varepsilon$ and $n$ be as in (2). Then

$$
n<B(\varepsilon):=\frac{\log \varepsilon}{\log (\sqrt[7]{\sqrt{D} / 4+290}-9 / 7)} .
$$

Although his result is correct, Nakamula's proof contains some misprints (see $\S 3)$ and besides, the argument is not sufficiently transparent to enable the reader to make the necessary adjustments. The object of the present paper is to give a different proof, making extensive use of computer algebra methods-in fact the Macintosh SE implementation of Maple, version 4.2.1 (see [1]) is used-of the following theorem:

Theorem 2. Let $\varepsilon$ and $n$ be as in (2). Then

$$
n<\widetilde{B}(\varepsilon):=\frac{\log \varepsilon}{\log (\sqrt[7]{\sqrt{D} / 4+76}-6 / 7)}
$$

It is an easy exercise to show that the upper bound $\widetilde{B}(\varepsilon)$ improves Nakamula's bound $B(\varepsilon)$. Indeed, on putting

$$
\lambda:=\sqrt[7]{\sqrt{D} / 4+76} \text { and } \mu:=\sqrt[7]{\sqrt{D} / 4+290}
$$

it follows that $\lambda>2$ and $\mu>\frac{9}{4}$, as the smallest possible discriminant $D$ equals 66125 (see [2, p. 244]). Application of these inequalities yields

$$
214=\mu^{7}-\lambda^{7}=(\mu-\lambda) \sum_{i=0}^{6} \mu^{i} \lambda^{6-i}>(\mu-\lambda) \frac{2685817}{4096}
$$

and hence $\mu-\lambda<\frac{3}{7}$, from which the assertion immediately follows. 
The real improvement of $\widetilde{B}(\varepsilon)$ over $B(\varepsilon)$ lies in the reduction of the fraction $\frac{9}{7}$ to $\frac{6}{7}$. How substantial this improvement can be for relatively small discriminants is clear from the table in $\S 5$. For large discriminants it is not immediately clear whether $\widetilde{B}(\varepsilon)$ always induces a better integer bound than $B(\varepsilon)$.

\section{THE DISCRIMINANT OF A RELATIVE UNIT}

Let $\varepsilon$ be a relative unit, that is, $\varepsilon \in H$ as in (1). If the conjugates of $\varepsilon$ with respect to $K / K_{3}$ are $\varepsilon$ and $\varepsilon^{\prime}$, then $\varepsilon \varepsilon^{\prime}=1$, and hence $\varepsilon^{\prime}=\varepsilon^{-1}$. This shows that we may assume $\varepsilon>1$, which we shall do from now on. With respect to $\mathbf{K} / \mathbf{K}_{2}$, let $\varepsilon, \varepsilon^{\prime \prime}$, and $\varepsilon^{\prime \prime \prime}$ be the conjugates of $\varepsilon$, so that $\varepsilon \varepsilon^{\prime \prime} \varepsilon^{\prime \prime \prime}=1$. As $\mathbf{K}_{3}$ is not totally real, $\varepsilon^{\prime \prime}$ and $\varepsilon^{\prime \prime \prime}$ are complex conjugates. All this implies that the field conjugates of $\varepsilon$ are

$$
\varepsilon, \frac{1}{\varepsilon}, \sqrt{\varepsilon} \exp (i \phi), \frac{1}{\sqrt{\varepsilon}} \exp (i \phi), \sqrt{\varepsilon} \exp (-i \phi), \frac{1}{\sqrt{\varepsilon}} \exp (-i \phi),
$$

for some $\phi$ with $0<\phi<\pi$. Further, let $\theta:=\operatorname{Tr}_{\mathbf{K} / \mathbf{K}_{3}}(\varepsilon), \theta^{\prime \prime}:=\operatorname{Tr}_{\mathbf{K} / \mathbf{K}_{3}}\left(\varepsilon^{\prime \prime}\right)$, and let $\theta^{\prime \prime \prime}$ be the complex conjugate of $\theta^{\prime \prime}$. Then

$$
\begin{gathered}
\left(x^{2}-\theta x+1\right)\left(x^{2}-\theta^{\prime \prime} x+1\right)\left(x^{2}-\theta^{\prime \prime \prime} x+1\right) \\
=x^{6}-s x^{5}+t x^{4}-u x^{3}+t x^{2}-s x+1,
\end{gathered}
$$

with $s, t, u \in \mathbf{Z}$, is the form of the minimal polynomial of $\varepsilon$ over $\mathbf{Q}$. On setting

$$
a:=\sqrt{\varepsilon}+1 / \sqrt{\varepsilon},
$$

we deduce from (3) and (4) that

$$
\begin{aligned}
& s=\theta+2 a \cos \phi, \\
& t=1+\theta(1+s-\theta)+a^{-2}(s-\theta)^{2} .
\end{aligned}
$$

A relation between $s, t$, and $u$ is quickly established as follows. On putting $\alpha:=\operatorname{Tr}_{\mathbf{K} / \mathbf{K}_{2}}(\varepsilon)$ and $\alpha^{\prime}:=\operatorname{Tr}_{\mathbf{K} / \mathbf{K}_{2}}\left(\varepsilon^{\prime}\right)$, we find that the minimal polynomial of $\varepsilon$ is also given by

$$
\left(x^{3}-\alpha x^{2}+\alpha^{\prime} x-1\right)\left(x^{3}-\alpha^{\prime} x^{2}+\alpha x-1\right),
$$

so that

$$
s=\alpha+\alpha^{\prime}, \quad t=\alpha+\alpha^{\prime}+\alpha \alpha^{\prime}, \quad u=2+\alpha^{2}+\alpha^{\prime 2},
$$

from which we immediately deduce the relation $u=s^{2}+2 s-2 t+2$. Hence, the minimal polynomial of $\varepsilon$ is

$$
x^{6}-s x^{5}+t x^{4}-\left(s^{2}+2 s-2 t+2\right) x^{3}+t x^{2}-s x+1, \quad s, t \in \mathbf{Z} .
$$

Finally, it is now rather easy to express the discriminant $D(\varepsilon)$ with respect to $\mathbf{K} / \mathbf{Q}$ in terms of $\varepsilon$ and $\phi$. If

$$
F(x):=\left(a^{3}-3 a-x\right)^{2}(a-x)^{3}(a+x)\left(4-x^{2}\right), \quad x \in \mathbf{R},
$$

then we have

$$
\sqrt{D(\varepsilon)}=\left(\varepsilon-\varepsilon^{-1}\right)(\alpha-2) F(2 \cos \phi) .
$$

Note that $D(\varepsilon)>0$, as $\mathbf{K}=\mathbf{Q}(\varepsilon)$ has an even number of complex conjugate pairs. Because of (9), it goes without saying that we are only interested in the range $(-2,2)$ of arguments of the function $F$. 
If for all $a>2, U_{F}(a)$ is an upper bound for the function $F$ on $(-2,2)$, then

$$
\sqrt{D(\varepsilon)} \leq a U_{F}(a) \sqrt{\left(a^{2}-4\right)^{3}}
$$

Hence, in order to find an upper bound for the discriminant $D(\varepsilon)$ in terms of $\varepsilon$ or $a$, it is sufficient to find one for the function $F$ on the interval $(-2,2)$.

\section{UPPER BOUND FOR THE FUNCTION $F$}

The function $F$ is a polynomial in $x$ of degree 8 , the coefficients of which are polynomials in $a$. Hence, solving $F^{\prime}(x)=0$ analytically-in order to find stationary points-is either trivial (which is not the case here) or impossible, the more so because a parameter $a$ is involved. Now Nakamula in the paper cited gets around this problem by estimating the stationary point in terms of $a$. However, the phrase "after a tedious calculation" does not give any insight into what really happens. Moreover, there are a few misprints in the proof. To be more precise, on p. 231 of [2], the constant term in the definition of $A_{3}$ should be -104 instead of -108 , and the coefficient of $\gamma^{-3}$ in the closing line should be +1280 instead of -1280 . We shall proceed in a different way.

First of all, as $a>2$ and hence $a^{3}-3 a>2$, the function $F$ does not vanish on the interval $(-2,2)$. Further, as $F(0)>0, F$ must have a unique and positive maximum $M_{F}(a)$ at $x=x(a)$ on $(-2,2)$ for all $a>2$.

The general approach we plan to adopt, and which ultimately leads to an upper bound for $M_{F}$, may be described as follows.

Main procedure. For every $x$-interval $I \subset(-2,2)$ containing $x(a)$ and on which $F$ is a concave function, the graph of $F$ lies entirely below the tangent to the graph at any point with $x$-coordinate belonging to $I$. Now take two points in $I$, one to the left and one to the right of $x(a)$. Then the tangents to the graph of $F$ at the corresponding points on the graph intersect in a point with $x$-coordinate $\geq M_{F}$.

So we have to determine a suitable subinterval $I$ of $(-2,2)$ and suitable points sufficiently close to $x(a)$ to the left and right of $x(a)$ to make the process work. By inspection, $F^{\prime}(-1)>0$ and $F^{\prime}\left(-\frac{2}{a}\right)<0$ (see the list of $F^{\prime}$-values in [3]), so that $x(a)$ belongs to the interval $\left(-1,-\frac{2}{a}\right)$ for all $a>2$. However, as $\lim _{a \rightarrow \infty} F^{\prime}(-1) / a^{10}=2$ and $\lim _{a \rightarrow \infty} F^{\prime}\left(-\frac{2}{a}\right) / a^{10}=0$, the value -1 is a rather bad choice for large values of $a$; the left endpoint $-\frac{4}{a}$ apparently is a better choice. Unfortunately, for values of $a$ close to 2 , the function $F$ is not concave on the corresponding interval $\left(-\frac{4}{a},-\frac{2}{a}\right)$. For instance, if $a=2$ and $-2<x<-1-\frac{1}{7} \sqrt{21}$, then $F^{\prime \prime}(x)>0$. As the sign of $F^{\prime \prime}$ cannot be determined by mere inspection, we proceed by attempting to locate all real zeros of this polynomial function. As it turns out, all six zeros of $F^{\prime \prime}$ are real, amongst which the simple zero $x=a$. Now, by determining all sign changes, we shall know approximately where $F^{\prime \prime}$ takes negative values. Let $\bar{F}$ be the polynomial of degree 5 over $\mathbf{R}$ with

$$
\bar{F}(x)=\frac{F^{\prime \prime}(x)}{a-x} \quad \text { for } x \neq a .
$$

Clearly, the leading coefficient of $\bar{F}(x)$ is -1 , and our table of signs indicates that $\bar{F}$ does not change sign on the intervals $\left(-1,-\frac{2}{a}\right)$ and $\left(-\frac{3}{a},-\frac{2}{a}\right)$. Indeed, 


\begin{tabular}{|c|c|c|c|c|c|c|c|}
\hline \multicolumn{8}{|c|}{$\begin{array}{c}\text { The sign of the function } \bar{F} \\
s(x):=\text { sign of } \bar{F}(x) ; p(a):=\frac{3}{4} a^{3}-a^{2}+a-2\end{array}$} \\
\hline$x$ & $-\infty<$ & -1 and $-\frac{3}{a}$ & $-\frac{2}{a}$ & & & $p(a)$ & $<\infty$ \\
\hline$s(x)$ & + & - & - & & & + & - \\
\hline
\end{tabular}

the position of every one of the five zeros of $\bar{F}$ is accounted for outside these intervals. Note that

$$
\max \left(-1,-\frac{3}{a}\right)<-\frac{2}{a}<\frac{a}{2}<a<p(a)=\frac{3}{4} a^{3}-a^{2}+a-2
$$

for $a>2$.

It is not easy to prove that the sign of $\bar{F}(x)$ at each of the six given $x$-values of the table is as indicated. The reason is that for certain rational functions of $a$ it needs to be established that no sign change occurs as $a$ ranges through $(2, \infty)$. Here the power of symbolic computation is needed for the first time. What we do is this. We simply substitute in $\bar{F}(x)$ for $x$ the relevant value expressed in terms of $a$, followed by the substitution of $a=b+2$. Of the resulting rational function of $b$, the numerator decides its sign, as the denominator is trivially positive for $b>0$. As it turns out, all nonzero coefficients are of equal sign, so that no sign change occurs in the range $b>0$. The explicit polynomials are printed in [3].

The following lemma now easily follows.

Lemma. Let $F$, defined as in (8), attain its unique maximum at $x(a) \in(-2,2)$. Further, let $I_{3}(a):=\left(-\frac{3}{a},-\frac{2}{a}\right)$ and $I_{4}(a):=\left(-\frac{4}{a},-\frac{2}{a}\right)$. Then

(i) $x(a) \in I_{3}(a)$ and $F$ is concave on $I_{3}(a)$ if $2<a \leq 4$,

(ii) $x(a) \in I_{4}(a)$ and $F$ is concave on $I_{4}(a)$ if $a>4$.

The concavity of the function $F$ in both cases has been established above. To show that $x(a)$ belongs to the relevant interval, it is sufficient to prove that $F^{\prime}\left(-\frac{3}{a}\right)>0$ if $2<a \leq 4$ and $F^{\prime}\left(-\frac{4}{a}\right)>0$ if $a>4$, as we already know that $F^{\prime}\left(-\frac{2}{a}\right)<0$. These assertions follow from the $F^{\prime}$-values as given in [3]. Note that $F^{\prime}\left(-\frac{3}{a}\right)>0$ in the range $a>2$ only if $a^{8}-32 a^{6}+174 a^{4}-378 a^{2}+324<0$, and the positive real zeros of this polynomial are 1.61662706 and 5.07997821, approximately.

To continue the main process, select $I=I_{3}(a)$ or $I=I_{4}(a)$, and calculate an upper bound $U_{F}(a)$ for $F$ on $I$ by intersecting the tangents to the graph of $F$ at the points corresponding to the endpoints of $I$. It is clear that $U_{F}(a)$ is a rational function of $a$. Note that, owing to the choice of endpoints, $U_{F}(a)$ is in fact a rational function of $a^{2}$. In the next section we shall construct an upper bound for $\sqrt{D(\varepsilon)}$ by means of (10), for both choices of $I$.

\section{Symbolic computations}

In this section we shall finalize the proof of Theorem 2 by manipulating polynomials with large rational coefficients and of rather high degree. Explicit information, including Maple programs, is provided in [3]. 
The right-hand side of inequality (10) is not a rational function of $a$. To restore rationality, we substitute for $a$ the expression $c+c^{-1}$; in fact we have $c:=\sqrt{\varepsilon}$, and then $a=c+c^{-1}$ by (5). Note that $c>1$ by assumption. Next we define polynomials $P, Q \in \mathbf{Z}[c]$ by

$$
\frac{P(c)}{Q(c)}:=U_{F}\left(c+c^{-1}\right)\left(c+c^{-1}\right)\left(c-c^{-1}\right)^{3},
$$

where the fraction is normalized. Then (10) yields

$$
\sqrt{D(\varepsilon)} \leq \frac{P(c)}{Q(c)} .
$$

As $U_{F}(a)$ is a rational function in the variable $a^{2}$, both $P(c)$ and $Q(c)$ are polynomials in $c^{2}$. For both choices of interval $I$ (see Lemma), the denominator $Q(c)$ has nonnegative integer coefficients, so that by the Euclidean algorithm,

$$
\frac{P(c)}{Q(c)}=M(c)+\frac{R(c)}{Q(c)}
$$

with $M, R \in \mathbf{Z}[c]$ and $\operatorname{deg}(R)<\operatorname{deg}(Q)$; it follows that for all $c>1$

$$
\frac{P(c)}{Q(c)} \leq M(c)+d_{1} \leq a_{M}\left(c^{2}+d_{2}\right)^{\operatorname{deg}(M) / 2}+d_{3},
$$

for suitable constants $d_{1}, d_{2}$, and $d_{3}$. Here, $a_{M}$ denotes the leading coefficient of $M(c)$. Our object is now to choose both $d_{2}$ and $d_{3}$ minimal, subject to inequality (11).

First case: $I_{3}(a)$. In case $I=I_{3}(a)=\left(-\frac{3}{a},-\frac{2}{a}\right)$, we have the polynomials

$$
\begin{aligned}
& P(c)=4 c^{74}+150 c^{72}+2370 c^{70}+20718 c^{68}+\cdots, \\
& Q(c)=c^{60}+32 c^{58}+420 c^{56}+2996 c^{54}+\cdots .
\end{aligned}
$$

Next consider (see (11))

$$
V(c):=-P(c)+Q(c)\left(4\left(c^{2}+d_{2}\right)^{7}+d_{3}\right)=\left(28 d_{2}-22\right) c^{72}+\cdots .
$$

The best possible choice for $d_{2}$ is such that the leading coefficient of $V(c)$ vanishes, i.e., $d_{2}=\frac{11}{14}$. Next substitute $c=1+b$ in the resulting expression for $V(c)$ and choose $d_{3}$ minimal and such that all coefficients of $V(1+b)$ as polynomials in $b$ are nonnegative. The optimal choice is now

$$
d_{3}=-\frac{6103515625}{26353376}=-231.602 \ldots
$$

which is obtained by setting $V(1)=0$, corresponding to $b=0$. Then

$$
V(1+b)=\frac{461}{7} b^{70}+4610 b^{69}+\frac{15805277}{98} b^{68}+\frac{184087458}{49} b^{67}+\cdots,
$$

the coefficients of which can have numerators as large as $10^{30}$. In [3] all coefficients of this polynomial are given.

The final inequality is

$$
\frac{P(c)}{Q(c)} \leq 4\left(c^{2}+\frac{11}{14}\right)^{7}-231
$$


which is true for all $c$ with $1<c \leq 2+\sqrt{3}$, as $2<a \leq 4$.

Second case: $I_{4}(a)$. Now we have $I=I_{4}(a)=\left(-\frac{4}{a},-\frac{2}{a}\right)$, and the corresponding polynomials are

$$
\begin{aligned}
& P(c)=4 c^{70}+172 c^{68}+3084 c^{66}+30308 c^{64}+\cdots, \\
& Q(c)=c^{56}+37 c^{54}+553 c^{52}+4453 c^{50}+\cdots
\end{aligned}
$$

As before (see (12)),

$$
V(c):=-P(c)+Q(c)\left(4\left(c^{2}+d_{2}\right)^{7}+d_{3}\right)=\left(28 d_{2}-24\right) c^{68}+\cdots,
$$

and here the best choice for $d_{2}$ is $\frac{6}{7}$. Again, substitute $c=1+b$ in the resulting expression for $V(c)$ and choose $d_{3}$ such that all coefficients of $V(1+b)$ are nonnegative. The optimal choice is

$$
d_{3}=-\frac{250994068}{823543}=-304.773 \ldots,
$$

which again is obtained by setting $V(1)=0$. Then

$$
V(1+b)=\frac{544}{7} b^{66}+\frac{35904}{7} b^{65}+\frac{8322392}{49} b^{64}+\frac{184124928}{49} b^{63}+\cdots .
$$

As in the first case, the coefficients can have very large numerators. See [3] for full information.

The result is that

$$
\frac{P(c)}{Q(c)} \leq 4\left(c^{2}+\frac{6}{7}\right)^{7}-304
$$

for all $c>2+\sqrt{3}$, because here $a>4$.

It remains to combine both inequalities (13) and (14) and create a single inequality valid for all values of $a>2$. This is a straightforward matter. From

$$
\begin{aligned}
\left(c^{2}+\frac{6}{7}\right)^{7}-\left(c^{2}+\frac{11}{14}\right)^{7} & =\sum_{n=0}^{6}\left(\begin{array}{l}
7 \\
n
\end{array}\right)\left(c^{2}+\frac{11}{14}\right)^{n}\left(\frac{1}{14}\right)^{7-n} \\
& >\sum_{n=0}^{6}\left(\begin{array}{l}
7 \\
n
\end{array}\right)\left(1+\frac{11}{14}\right)^{n}\left(\frac{1}{14}\right)^{7-n} \\
& =\left(\frac{13}{7}\right)^{7}-\left(\frac{25}{14}\right)^{7}>\frac{73}{4},
\end{aligned}
$$

it follows that for all $c>1$

$$
4\left(c^{2}+\frac{6}{7}\right)^{7}-304>4\left(c^{2}+\frac{11}{14}\right)^{7}-231 .
$$

Hence, for all $a>2$

$$
D \leq D(\varepsilon) \leq 16\left(\left(c^{2}+\frac{6}{7}\right)^{7}-76\right)^{2},
$$

and Theorem 2 immediately follows.

\section{COMPARISON OF BOUNDS}

In this final section we shall give examples to demonstrate the extent to which the bound $\widetilde{B}(\varepsilon)$ is an improvement of $B(\varepsilon)$, the one given by Nakamula in [2]. 


\begin{tabular}{|c|c|c|c|c|c|c|c|c|}
\hline \multicolumn{9}{|c|}{$\begin{array}{c}\text { Comparing } B(\varepsilon) \text { and } \tilde{B}(\varepsilon) \\
\varepsilon \text { has minimal polynomial }(7) \text { with parameters } s \text { and } t \\
1.2(3) \text { is short for } 1.2 \times 10^{3} \\
D, D_{2} \text { and } D_{3} \text { are the discriminants of } \mathbf{K}, \mathbf{K}_{2} \text { and } \mathbf{K}_{3} \\
\text { Entry } n_{i} \text { in column } 1 \text { corresponds to Table } n \text {, line } i \\
\text { and }\left(^{*}\right) \text { corresponds to Example } 7.2 \text { (iii) of [2] }\end{array}$} \\
\hline$[2]$ & $s$ & $t$ & $D$ & $D_{2}$ & $-D_{3}$ & $\varepsilon$ & $B(\varepsilon)$ & $\tilde{B}(\varepsilon)$ \\
\hline 37 & -1 & -9 & 1760913 & 33 & 231 & $3.4(0)$ & 6.2 & 3.0 \\
\hline $3_{3}$ & -1 & -8 & 390224 & 29 & 116 & $3.3(0)$ & 11.9 & 4.3 \\
\hline $2_{3}$ & -1 & -6 & 7260624 & 21 & 588 & $2.9(0)$ & 3.3 & 2.0 \\
\hline $3_{2}$ & -1 & -4 & 140608 & 13 & 104 & $2.4(0)$ & 16.9 & 4.4 \\
\hline $3_{25}$ & 0 & -2 & 1782272 & 8 & 472 & $2.2(0)$ & 4.0 & 2.0 \\
\hline 39 & 1 & -14 & 3631696 & 61 & 244 & $5.1(0)$ & 6.3 & 3.4 \\
\hline $3_{1}$ & 1 & -6 & 219501 & 29 & 87 & $3.8(0)$ & 18.9 & 5.7 \\
\hline $3_{12}$ & 1 & -3 & 1105425 & 17 & 255 & $3.2(0)$ & 7.0 & 3.1 \\
\hline $2{ }_{1}$ & 1 & 0 & 450000 & 5 & 300 & $2.1(0)$ & 7.0 & 2.6 \\
\hline $3_{23}$ & 2 & -37 & 7744000 & 40 & 440 & $8.0(0)$ & 6.3 & 3.8 \\
\hline 27 & 2 & -9 & 4320000 & 12 & 300 & $5.0(0)$ & 5.8 & 3.3 \\
\hline $3_{4}$ & 2 & -5 & 184832 & 8 & 152 & $4.3(0)$ & 22.9 & 6.6 \\
\hline $3_{5}$ & 5 & -2 & 2382032 & 53 & 212 & $6.3(0)$ & 8.3 & 4.2 \\
\hline $3_{20}$ & 7 & -8 & 20720464 & 109 & 436 & $8.9(0)$ & 5.1 & 3.4 \\
\hline $3_{16}$ & 10 & -78 & 12986073 & 113 & 339 & $1.6(1)$ & 7.3 & 4.6 \\
\hline $3_{19}$ & 19 & 96 & 9528128 & 53 & 424 & $1.3(1)$ & 7.2 & 4.5 \\
\hline $3_{6}$ & 22 & -193 & 1120581 & 21 & 231 & $3.0(1)$ & 20.4 & 9.2 \\
\hline $3_{10}$ & 22 & -65 & 793117 & 13 & 247 & $2.6(1)$ & 22.8 & 9.6 \\
\hline $3_{18}$ & 22 & 6 & 23142177 & 137 & 411 & $2.3(1)$ & 7.1 & 4.8 \\
\hline $3_{11}$ & 22 & 63 & 325125 & 5 & 255 & $2.0(1)$ & 33.2 & 11.3 \\
\hline $3_{21}$ & 23 & 124 & 968000 & 5 & 440 & $1.7(1)$ & 18.1 & 8.0 \\
\hline 38 & 25 & 162 & 4108797 & 77 & 231 & $1.6(1)$ & 10.3 & 5.8 \\
\hline $2_{10}$ & 28 & 191 & 69574032 & 33 & 1452 & $1.9(1)$ & 5.3 & 3.9 \\
\hline $3_{24}$ & 30 & -401 & 8339441 & 41 & 451 & $4.1(1)$ & 11.0 & 6.7 \\
\hline$(*)$ & 38 & 319 & 66125 & 5 & 23 & $2.8(1)$ & 122.8 & 21.2 \\
\hline $2_{2}$ & 42 & 375 & 559872 & 12 & 108 & $3.1(1)$ & 28.6 & 11.2 \\
\hline $3_{13}$ & 49 & 458 & 5527125 & 85 & 255 & $3.8(1)$ & 12.2 & 7.1 \\
\hline $3_{14}$ & 81 & -486 & 11655261 & 109 & 327 & $8.8(1)$ & 12.1 & 7.6 \\
\hline $3_{15}$ & 86 & -65 & 561125 & 5 & 335 & $8.8(1)$ & 37.2 & 14.6 \\
\hline $3_{17}$ & 110 & -2561 & 11279504 & 89 & 356 & $1.3(2)$ & 13.3 & 8.4 \\
\hline 24 & 141 & -3090 & 2278125 & 5 & 675 & $1.6(2)$ & 23.1 & 11.8 \\
\hline $2_{5}$ & 142 & 2735 & 12778713 & 17 & 867 & $1.2(2)$ & 12.6 & 8.0 \\
\hline $2_{6}$ & 158 & 2751 & 66854673 & 57 & 1083 & $1.4(2)$ & 8.9 & 6.5 \\
\hline $3_{22}$ & 266 & -9893 & 1548800 & 8 & 440 & $3.0(2)$ & 30.1 & 14.4 \\
\hline 29 & 1581 & -111810 & 36756909 & 21 & 1323 & $1.6(3)$ & 15.1 & 10.6 \\
\hline 28 & 1666 & 59015 & 21600000 & 60 & 300 & $1.6(3)$ & 17.0 & 11.4 \\
\hline
\end{tabular}


It seems fair to choose examples similar to those provided there. In fact, we have chosen exactly the same $(s, t)$-values as those offered in the examples and in Tables 2 and 3 of the paper cited. The first column of the table gives a reference to Nakamula's tables and examples. We noticed a couple of misprints in these tables: the first entry of the second line of Table 2 should be 80 instead of 60 and the third entry of line 4 of Table 3 should be -8 and not 8 .

\section{ACKNOWLEDGMENT}

The author gratefully acknowledges the referee's clarification of Nakamula's argument, pointing out the actual misprints on p. 231 of [2].

\section{BIBLIOGRAPHY}

1. Macintosh Maple 4.2.1, Symbolic Computation Group, Department of Computer Science, Univ. of Waterloo, 1990.

2. Ken Nakamula, Class number calculation of a sextic field from the elliptic unit, Acta Arith. 45 (1985), 230-247.

3. R. J. Stroeker, Appendix to improvement of Nakamula's upper bound for the absolute discriminant of a sextic number field with two real conjugates, Econometric Inst., Erasmus Univ. Rotterdam, Report Series 9069/B, 1990.

Econometric Institute, Erasmus University RotTerdam, P.O. Box 1738, 3000 DR RotTERDAM, THE NETHERLANDS

E-mail address: stroeker@cvx.eur.nl.bitnet 